\title{
A Simulation Based Investigation of High Latency Space Systems Operations
}

\author{
Zu Qun Li \\ NASA Johnson Space Center \\ 2101 NASA Road 1 \\ Houston, TX 77058 \\ zuqun.li@nasa.gov
}

\author{
Michael Moore \\ CACI International Inc \\ NASA Johnson Space Center \\ Houston, TX 77058 \\ guy.decarufel@nasa.gov \\ Paul Bielski \\ NASA Johnson Space Center \\ 2101 NASA Road 1 \\ Houston, TX 77058 \\ paul.bielski@nasa.gov
}

\author{
Edwin Z. Crues \\ NASA Johnson Space Center \\ 2101 NASA Road 1 \\ Houston, TX 77058 \\ edwin.z.crues@nasa.gov
}

\begin{abstract}
This study was the first in a series of planned tests to use physics-based subsystem simulations to investigate the interactions between a spacecraft's crew and a ground-based mission control center for vehicle subsystem operations across long communication delays. The simulation models the life support system of a deep space habitat. It contains models of an environmental control and life support system, an electrical power system, an active thermal control systems, and crew metabolic functions. The simulation has three interfaces: 1) a real-time crew interface that can be use to monitor and control the subsystems; 2) a mission control center interface with data transport delays up to 15 minute each way; and 3) a real-time simulation test conductor interface used to insert subsystem malfunctions and observe the interactions between the crew, ground, and simulated vehicle.
\end{abstract}

The study was conducted at the 21st NASA Extreme Environment Mission Operations (NEEMO) mission. The NEEMO crew and ground support team performed a number of relevant deep space mission scenarios that included both nominal activities and activities with system malfunctions. While this initial test sequence was focused on test infrastructure and procedures development, the data collected in the study already indicate that long communication delays have notable impacts on the operation of deep space systems.

For future human missions beyond cis-lunar, NASA will need to design systems and support tools to meet these challenges. These will be used to train the crew to handle critical malfunctions on their own, to predict malfunctions and assist with vehicle operations. Subsequent more detailed and involved studies will be conducted to continue advancing NASA's understanding of space systems operations across long communications delays.

\section{TABLE OF CONTENTS}

1. INTRODUCTION.................................

2. SubSYSTEM Simulation DEVELOPMENT ........1

3. SubSystem Simulation Study at NEEMO 21 .. 5

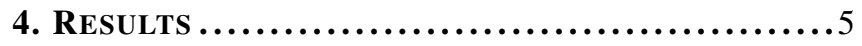

5. Conclusion ................................6

ACKNOWLEDGMENTS ..............................7

REFERENCES .....................................7

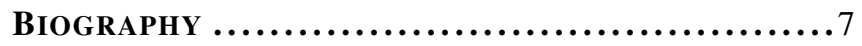

\section{INTRODUCTION}

NASA's human space program has developed considerable experience with near Earth space operations. Although NASA has experience with deep space robotic missions, NASA has little substantive experience with human deep space operations. Even in the Apollo program, the missions lasted only a few weeks and the communication latencies were on the order of seconds. Human missions beyond the relatively close confines of the Earth-Moon system will involve durations measured in months and communications latencies measured in minutes. To minimize crew risk and to maximize mission success, NASA needs to develop a better understanding of the implications of these types of mission durations and communication latencies on vehicle design, mission design and flight controller interaction with the crew.

To begin to address these needs, NASA performed a study using a physics-based subsystem simulation to investigate the interactions between spacecraft crew and a groundbased mission control center for vehicle subsystem operations across long communication delays. The study was conducted at the 21st NASA Extreme Environment Mission Operations (NEEMO) mission between July 21st and Aug 5th of year 2016. The NEEMO mission provides ideal conditions for this study with crew in the loop, an active control center, and realtime flow of high latency communications and data. NEEMO crew and ground support personnel worked together through procedures including activation of the habitat power system, opening the hatch between the habitat and a visiting vehicle, transferring simulated crew members between vehicles, overcoming subsystem malfunctions, and other housekeeping activities.

\section{Subsystem Simulation DeVelopment}

To support this study, the NASA Exploration Systems Simulations (NExSyS) team at NASA's Johnson Space Center (JSC) developed an integrated simulation of the subsystems of a deep space habitat. This paper addresses three important aspects of this simulation development: tools, models and interfaces. The tool section discusses two principal inhouse simulations packages used in the development: the Trick Simulation Environment and the General Use Nodal Network Solver (GUNNS). The models section discusses the subsystem models developed for the simulation: power, thermal control, life support, and crew metabolic functions. The subsystems modeling also includes subsystem specific 
malfunctions used to represent challenging contingency situations requiring cooperation between crew and the mission control center. The interface section discusses the separation and content of the three principal simulation interfaces used during the study: crew, mission control and test conductor.

\section{Simulation Tools}

Many software packages and tools were used in the development of the simulation used in this study. However, there are two principal packages that warrant discussion: Trick and GUNNS.

Trick is a NASA Open Source simulation framework for developing physics-based simulations. It provides many features such as real-time synchronization, job scheduling, simulations state save and restore, data recording, runtime variable manipulation, numerical integration, simulation event management, and Monte Carlo simulation execution. Trick can also work with external software such as Input Device Framework (IDF), which is another Open Source software developed at JSC, to develop human-in-the-loop simulation for crew training [1].

GUNNS is a software package uses to model flow systems in a time based simulation. It uses basic nodal analysis techniques to simulate fluid, electrical, and thermal systems. GUNNS also supports interconnecting these systems across user defined boundaries in order to simulate the real world interaction between separate systems. GUNNS is not intended as a stand in for more specialized tools that provide high fidelity simulation for specific aspects. It is considered to be a medium-fidelity model that is mainly uses for training simulations and high level system performance analysis. GUNNS was developed with $\mathrm{C}++$ object oriented design to maximize its re-usability. Models such as batteries, pipes, valves, and solar panels are combined in networks to represent the physical system. The simulation developer can also use a GUI called GunnShow to create system models in a drag-and-drop fashion. Figure 1 is an example of fluid network developed using GunnShow. [2]

\section{Subsystem Simulation Model}

The subsystem simulation models the environmental control and life support system (ECLSS), electrical power system (EPS), and thermal control system (TCS) of a deep space habitat. These models were first developed separately using GunnShow, and then integrated together to form a larger and more complex network. The habitat has an attached airlock and visiting vehicle to simulate one possible deep space mission scenario. Figure 2 shows the layout of the three habitable volumes. The simulation also contains component malfunction capabilities which can be use to simulate contingency scenarios. These scenarios pose a challenge for both the crew and the mission control to overcome. Diagnosing and resolving these malfunctions often requires cooperation between the crew and the mission control.

Environmental Control and Life Support System-ECLSS is responsible for monitoring and controlling the cabin atmosphere to be within the comfortable and safe zone for humans. ECLSS provides oxygen for metabolic consumption, provides water for crew consumption and usage, and maintains cabin temperature and humidity in the designed range of operation. The ECLSS model used in this simulation is based on previous work [3]. Figure 3 gives a high level overview of the ECLSS as modeled in the simulation.

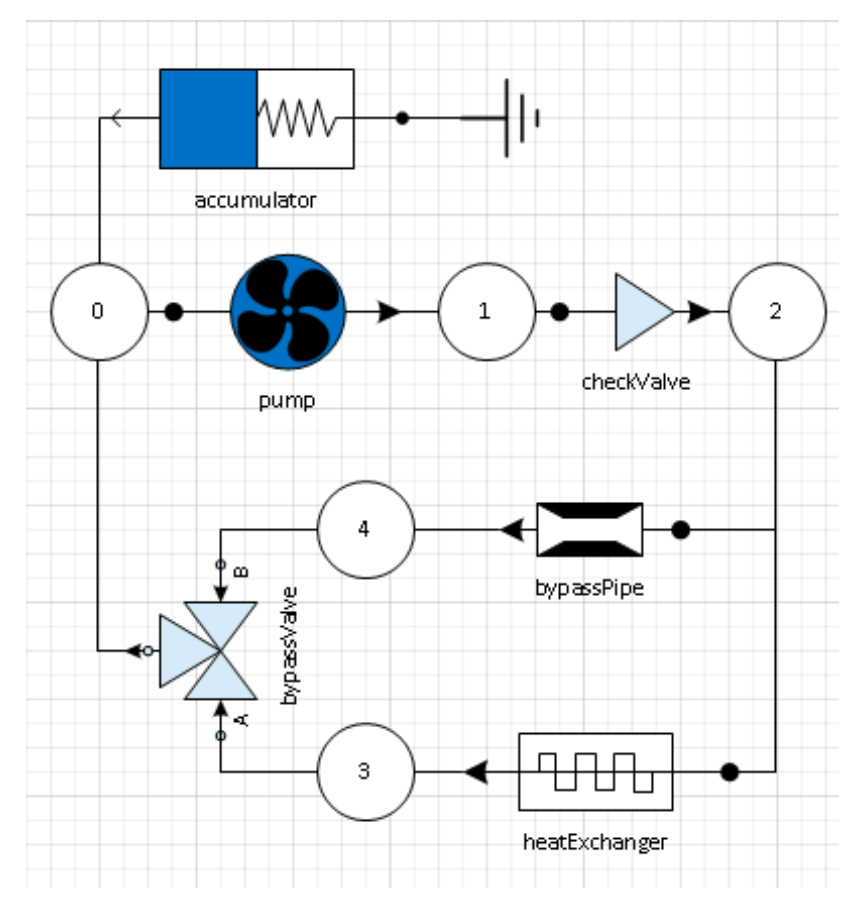

Figure 1. Example GunnShow Network.

Figure 3 shows the ECLSS model containing three major components: 1) Air Revitalization System (ARS); 2) Waste Processing System (WPS); 3) Water Recovery System (WRS) [3].

The ARS contains the following elements:

Trace Contaminant Control System (TCCS): removes hazardous trace contamination from the cabin air [4].

Condensing Heat Exchanger (CHx): controls cabin air humidity.

Carbon Dioxide Removal Assembly (CDRA): removes $\mathrm{CO}_{2}$ from the cabin air and is used in the Sabatier reactor for water recovery.

Solid Polymer Electrolysis (SPE): generates $\mathrm{O}_{2}$ and $\mathrm{H}_{2}$ from water. $\mathrm{O}_{2}$ flows into the $\mathrm{O}_{2}$ tank, and $\mathrm{H}_{2}$ is use in Sabatier reactor for water recovery.

Sabatier: a Sabatier reactor model that recovers water used in the SPE process.

Pressure Control System (PCS): monitors and maintain the cabin atmosphere inside $\mathrm{O}_{2}$ safe zone.

02 and N2 Tanks: $O_{2}$ and $N_{2}$ storage tanks.

The WPS contains following elements:

Waste Storage: solid waste storage

Heat Meld Compactor (HMC): recovers residual water from trash and compacts the trash to reduce its volume.

CHx: condenses water vapor for use in the WPA.

Brine Residual in Containment (BRIC): further extract water from brine or feces.

The WRS contains following elements:

Pretreatment Storage: stores chemicals for treating the crew urine prior to processing it in the water recovery system.

UPIX: removes calcium from waste water. 


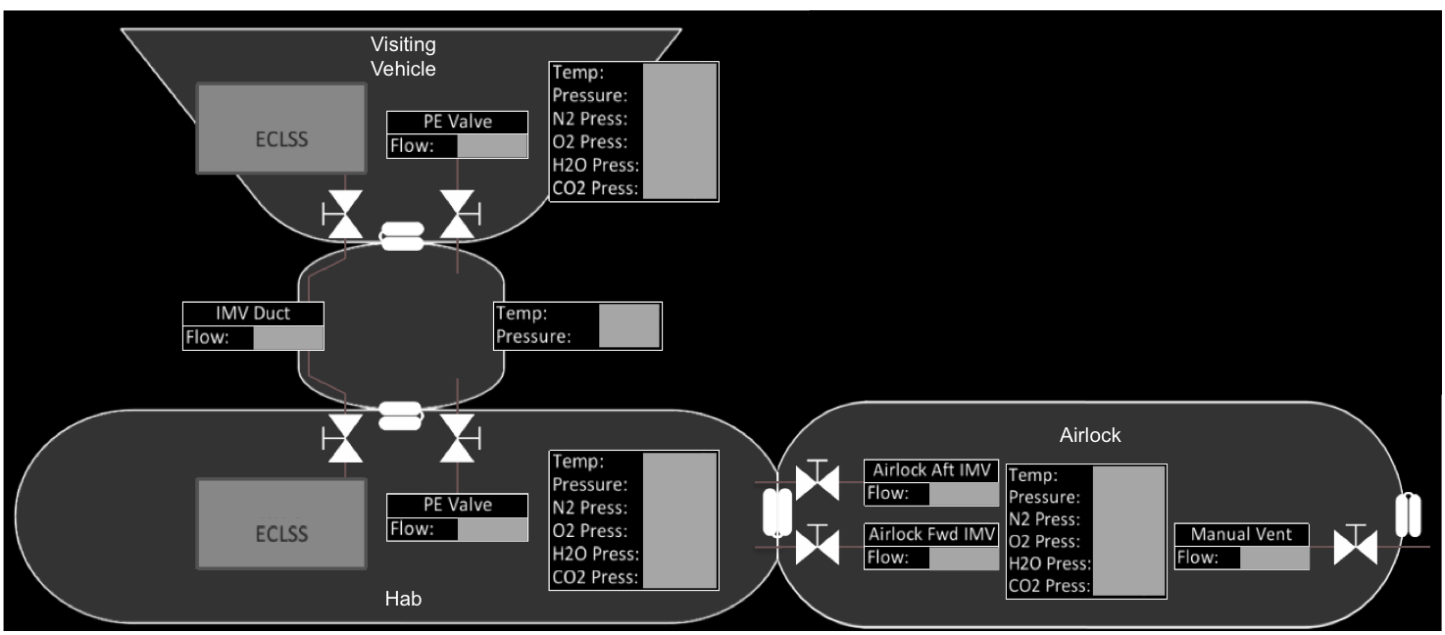

Figure 2. Overview of Subsystem Simulation Cabins

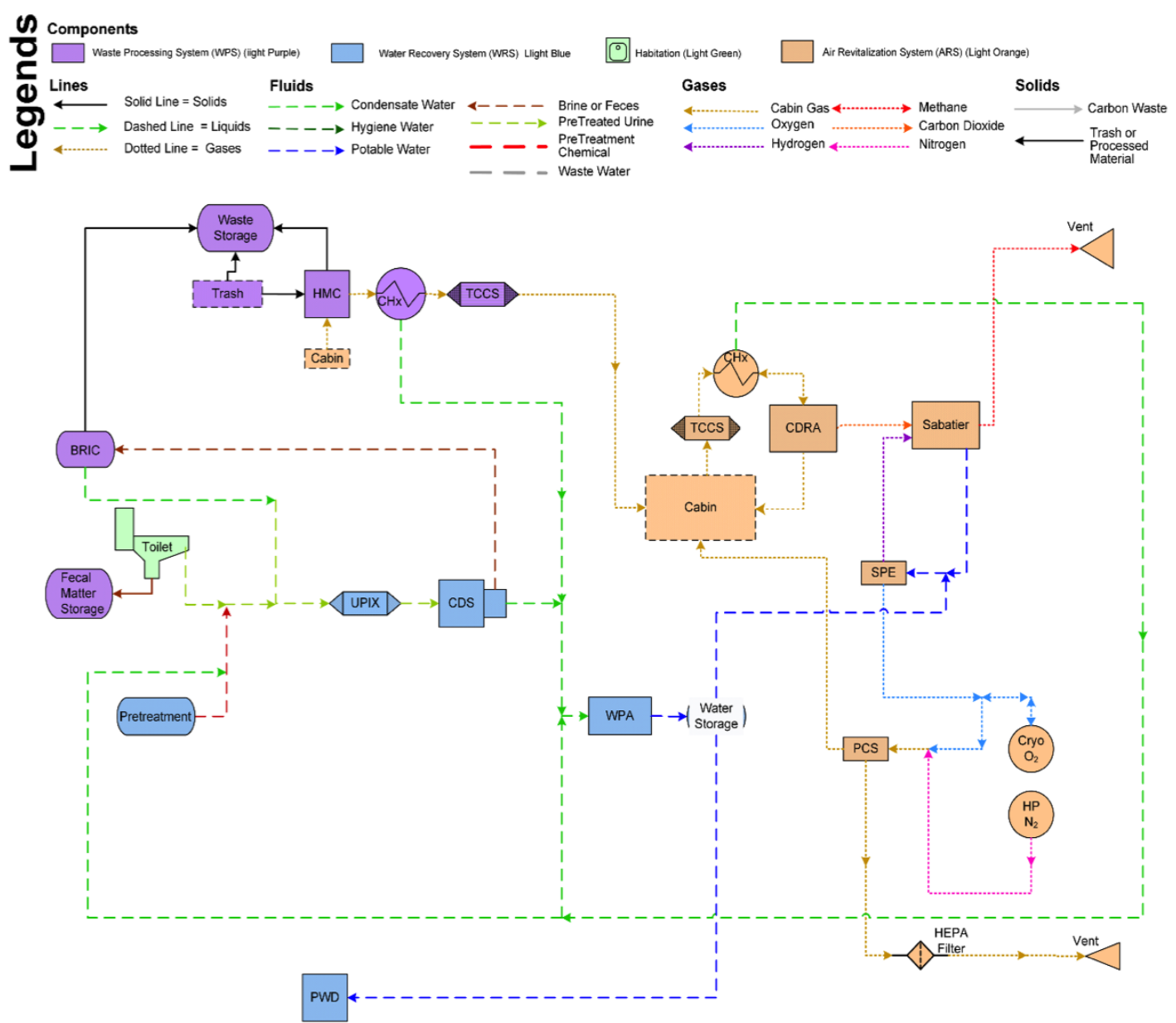

Figure 3. High Level Overview of ECLSS model

Cascade Distillation System (CDS): recovers water from waste water

Water Processor Assembly (WPA): produces potable water from distillate water

Potable Water Dispenser (PWD): manages distribution of potable water.
The performance of these models is based on operational data from similar systems in use on the International Space Station (ISS) or other relevant test data.

Thermal Control System-For the study, only the Active Thermal Control System (ATCS) is modeled for the TCS. Since neither the crew or mission control interact directly 
with the Passive Thermal Control System (PTCS), no PTCS was modeled for this study. The ATCS is modeled as three thermal control fluid loops. There is an Internal Thermal Control System (ITCS) loop that uses non-toxic working fluid to maintain the cabin and avionic temperature within the designed range. There are two External Thermal Control System (ETCS) loops used as primary active heat rejection system through body-mounted radiators. Both external loops will flow at all times during nominal operation. In case of failure in one loop, the malfunctioning external loop will be shut off and the un-compromised loop will increase its flow to compensate. ITCS and ECTS loops exchange their heat through a three-passage inter-loop heat exchanger.

Electrical Power System-The EPS model determines power usage over the simulated mission and includes electrical components that make major contributions to the power load. These include fans, liquid/gas separators, fluid pumps, heaters, and the SPE. The EPS also contains models of solar array panels as the primary power source and batteries as the secondary power source.

\section{Simulation Interfaces}

A principal objective of this study was to assess the effects of long communication latencies between crew in a deep space habitat and flight controllers in the Mission Control Center (MCC). This involves three principal roles in the study activity: crew, flight controllers and test conductors. Each role has a collection of displays (interfaces) that are relevant to their particular tasks (roles). The simulation infrastructure provides the relevant level of controllability and communication latency to the interfaces for each respective role. Figure 4 gives an overview of the interfaces: Crew Interfaces, Mission Control Center Support Interfaces, and Test Conductor Interfaces.

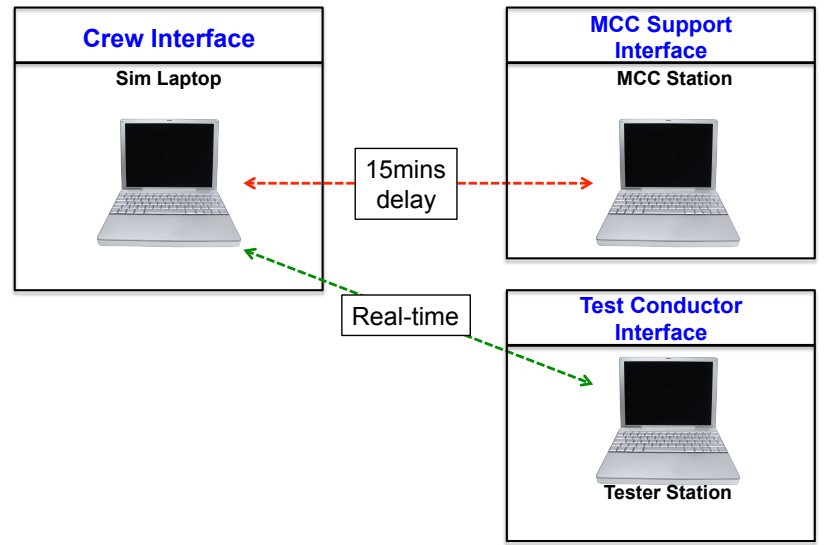

Figure 4. Simulation Interfaces Overview.

Note that the MCC support activities are delayed with respect to the crew but the test conductor's activities are not delayed. The delay represents the full end-to-end communication delay from light speed transmission time and communication pathway delays. This gives the test conductors the ability to assess the immediate activities of the crew and the associated affects on the deep space habitat's subsystems. It also gives the test conductors the ability to observer the activities of the MCC and the associated affects on the crew and deep space habitat's subsystems.

Crew Interface-The crew interfaces are real-time displays and controls for the deep space habitat's subsystems modeled in the simulation. These provide the crew with information on that current status of the systems and controls for those systems. These are presented to the crew as a series of interactive display pages. Figure 5 shows the status and control page for the Sabatier reactor that is part of the ARS.

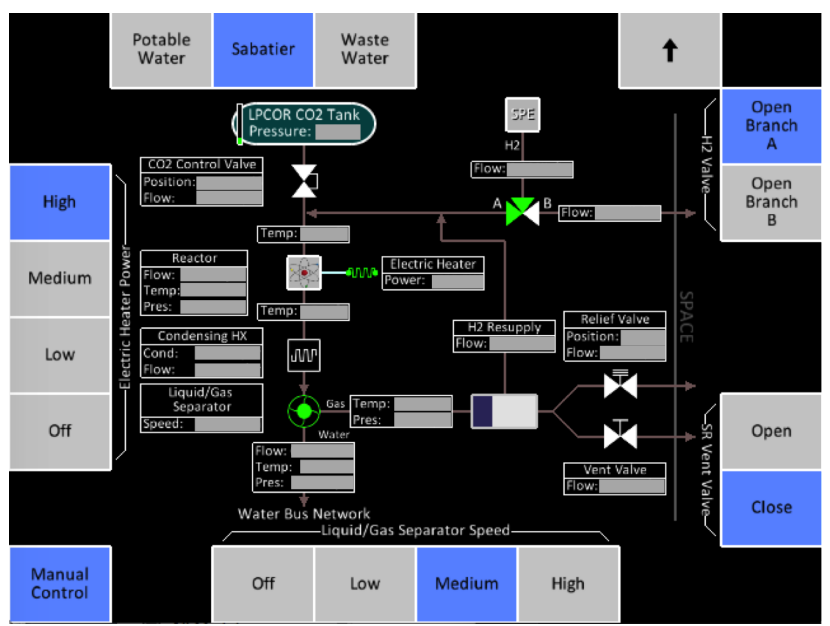

Figure 5. Crew/Test Conductor Interface.

Mission Control Center Support Interface-The MCC support interfaces are very similar to the crew displays (see Figure 6). However, all habitat information on the MCC displays is delayed by the communications latency between the deep space habitat and the MCC. In addition, the MCC displays have reduced capabilities as compared to the crew interfaces. For instance, the current implementation does not allow the MCC to command the habitat through these displays. Some commanding will be provided in subsequent studies.

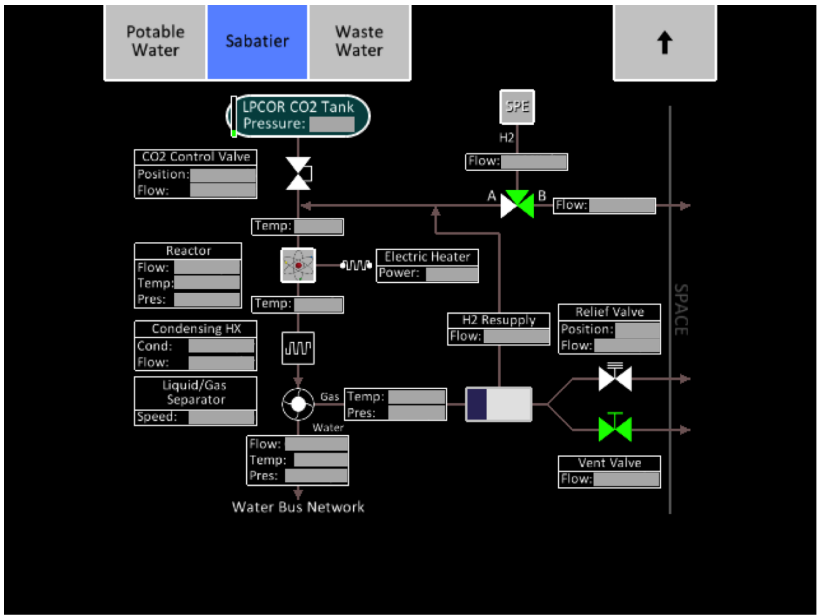

Figure 6. Mission Control Interface.

Test Conductor Interface-Like the crew interfaces, the test conductor's interfaces are also real-time displays and controls for the deep space habitat's subsystems modeled in the simulation (see Figure 5). In addition to these subsystem displays and controls, the test conductors are also provided a graphical interface into the simulation that allows the manipulation of simulation variables during runtime and the ability to insert subsystem malfunctions. Figure 7 shows the Trick variable server Trick View interface into the subsystem simulation. 


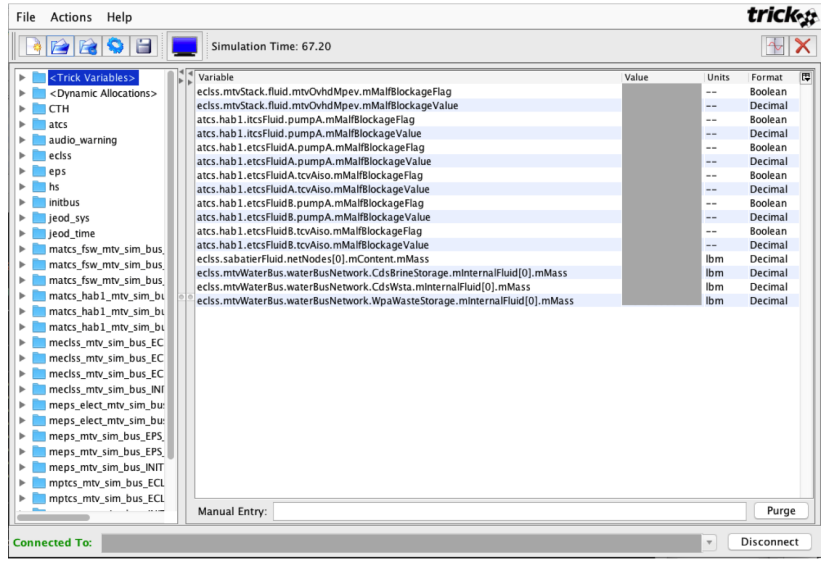

Figure 7. Test Conductor's Simulation Variable Interface.

\section{SubSystem Simulation STUdy at NEEMO 21}

NASA Extreme Environment Mission Operations (NEEMO) is an undersea mission conducted off the coast of Florida at the underwater research laboratory, Aquarius. Its purpose is to provide an analogue to space missions by putting crew members (aquanauts) in an extreme environment. Throughout the NEEMO mission, a group of astronauts, engineers and scientists lived in Aquarius for up to three weeks. During the mission, aquanauts were given a wide variety of tasks to perform. These tasks tend to focus on evaluating tools and techniques for future spacewalks on a variety of surfaces and gravity levels ranging from asteroids to the moons of Mars and the Martian surface [5]. In 2016, NEEMO simulated a mission to Mars with a 15 minute one-way communication delay between the Aquarius undersea habitat and the on-shore NEEMO mission control center. This provided a suitably representative environment in which to conduct the subsystem simulation studies investigating how crew members and mission control support teams work together to overcome system anomalies in the presence of long communication delays

Since this was the first deployment of the deep space habitat subsystem simulation, the study objectives were limited to deployment, infrastructure and simulation testing. Some principal objectives for this year's study were to improve understanding of the NEEMO operational environment, mission scheduling, network setup, and suitable hardware needed for more detailed and complex studies in subsequent years. Due to scheduling constraints for this particular NEEMO mission, the NEEMO crew members had only minimal training on the subsystem simulation. This limited the crew's ability to operate the system by themselves thus requiring contact with mission control center whenever a system anomaly occurred. In addition, limits in the MCC support staff for this NEEMO mission required the same person to act as both test conductor and mission control support for the study.

During the mission, the test conductor inserted malfunctions to the simulation without notifying the crew. An audio alarm sounded when the anomaly was detected in the subsystem. When the crew heard the audio alarm, they notified the mission control center about the situation and logged the malfunction into their mission log. After 15 minutes, mission control support received the message from the crew and started working on a solution for the malfunction. Once the
Table 1. Summary of subsystem simulation activity results at NEEMO 21

\begin{tabular}{|c|c|}
\hline Category & Time (minute) \\
\hline \hline Total time per activity & 69.35 \\
Communication delay & 36.00 \\
Response time & 5.37 \\
Action time & 10.35 \\
Task time & 10.41 \\
Other time & 7.22 \\
\hline
\end{tabular}

crew received the procedure from mission control (through another 15 minute communication delay), they started working on the problem. The crew notified mission control upon completion of the task.

At the early phase of the NEEMO mission, crew members went through a series of procedures to power on the habitat, open the hatch between visiting vehicle and the habitat, and transfer simulated crew members from the visiting vehicle into the habitat. After crew completed these nominal activities, the test conductor started inserting one malfunction for each mission day. These anomalies include malfunctions in the ITCS, ETCS, waste water processing system, oxygen generation system, and Sabatier reactor controller. Anomaly complexity ranged from simple to medium, where a simple malfunction normally requires one communication exchange while a medium malfunction normally requires two communication exchanges.

\section{RESULTS}

Five crew members participated in the study, and total of 8 activities/malfunctions were conducted during the mission. One out of the eight activities was conduced with real-time communication, the rest were done through delayed communication (15 minutes one-way). The first Subsystem activity was conducted on Mission Day (MD) 5, and the last activity was conducted on MD 13.

\section{Activity Result}

Excluding the one activity that was done through real-time communication and the two activities that have very long action times, the average amount of time spent on different categories are shown in Table 1, and Figure 8 shows the percentage of time spent on each category.

In Table 1, "Communication time" is the amount of time needed for the MCC and NEEMO Crew to communicate with each other. In this case, it is 15 minutes one-way. "Response time" is the amount of time required for the crew to log the malfunction into mission log and inform the MCC about the anomaly after the malfunction alarm was sounded. "Action time" is amount of time required for the crew to start working on the task after they received the instruction from the MCC. "Task time" is amount of time that crew spent on the task. "Other time" is amount of time for other action that includes time for the MCC to prepare and send the instruction.

The average total time per activity is about 69 minutes. Time for communication delay takes 36 minutes on average (there is one activity which required two communication exchanges), which is about $52 \%$ of the average total time 


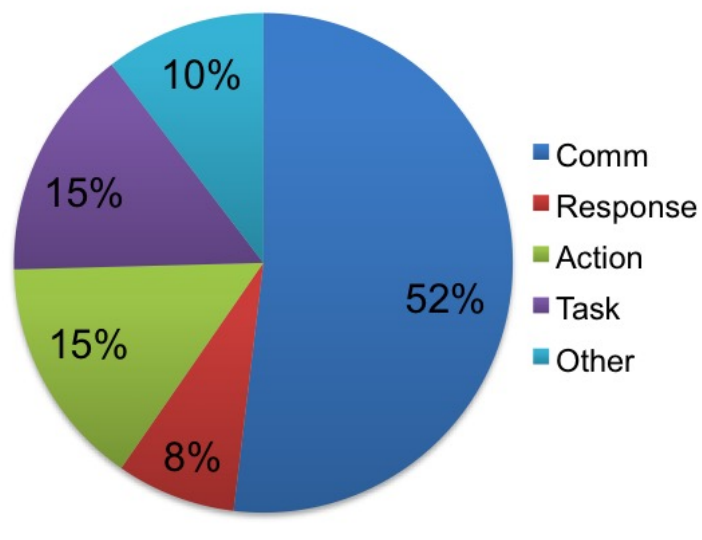

Figure 8. Percentage of Time Spent on each Category.

spent. As expected, when compared to the one activity that was done with zero time delay, long communication delays significantly increased malfunction reaction time. Clearly, communications delay is one of the more challenging aspects of sending human to Mars.

For missions in the Earth-Moon system, communication latencies are measured in milliseconds and seconds. This allows for near real-time interaction between ground based mission support and crew on the spacecraft. This permits a support model where the ground based MCC can identify potential malfunctions and assist the crew in overcoming any anomalies using short interactive exchanges of information. For deep space missions, like missions to Mars, every exchange of information comes with a time penalty. This requires a different MCC support model and greater autonomy for the crew in monitoring systems, maintaining systems and resolving critical malfunctions.

It is important to note that these deep space systems will be more complex and much further from Earth. The complexity of these systems and long communication delays make it impractical for the crew to constantly monitor the entire system and identify potential malfunctions in time for an Earth based resolution. This requires that the systems be highly reliable and easily maintained by the crew. The crew must also be trained to handle critical malfunctions by themselves. It is also important that systems monitoring tools are developed to assist the crew with routine systems operations and with the ability to predict malfunction. This predictive capability is critical in providing more time for MCC support in the presence of long communication delays. Subsystem simulations such as the one developed for this study will play a major role in future crew training and the development of tools to predict malfunction and assist with system operations.

\section{Feedback}

The NEEMO crew, the MCC support team and test conductor provided feedback at the conclusion of the NEEMO mission. This feedback is being used to assess the efficacy of the subsystem simulation and interfaces. It will also be used to direct improvements to the simulations, interfaces, infrastructure, and procedures used in subsequent studies. Crew members observed that vehicle and system malfunction could signif- icantly change the operations that they normally do in Low Earth Orbit with active engagement from MCC. The crew also identified specific hardware improvement, simulation display improvement, and training improvement. The test conductor and MCC support team identified an issue with poor connectivity between Aquarius and NEEMO mission control during high waves at sea. It was also noted that is was impractical for a single person to fill both roles of test conductor and MCC support team. These roles need to be separated in subsequent studies. All participants provided suggestions for improvements in training and scheduling. It was also suggested that the test conductor role could be performed from JSC with modifications to the current NEEMO communications and test infrastructure.

\section{Future Plans}

For future NEEMO based subsystem simulation studies, the simulation will be running on a simulation laptop in the Aquarius networks and all three interfaces will connect to it. The crew interface will be on a touch screen device that is on the same network (Aquarius network) as the simulation laptop. This way, when Aquarius network loses connection with the mission control center, the crew will still be able to monitor and control the subsystem simulation. The MCC interface will be in the NEEMO mission control center, and the test conductor interface will be at JSC. This way, MCC support and test conduction will be completely separate from each other, and the test conductor can insert malfunctions into the simulation at any time during the mission.

Future malfunction will be more complicated, and there will be critical malfunctions that requires immediate action from the crew. Therefore, more training will be provided to the crew so that they are able to operate the system and solve some critical malfunctions by themselves.

A more detailed and involved subsystem simulation study in the future will: further improve our understanding of the difference between LEO operations and high latency operations; identify challenges associate with high latency system operations; assist vehicle subsystem design for future mission beyond cis-lunar; and assist the development of intelligent onboard subsystem operation tools that can be use to identify potential system malfunctions in advance.

\section{Conclusion}

Long communication delays are one of the more challenging aspects of sending humans to Mars. This study uses an integrated subsystem simulation to expand our understanding of the difference between low latency operations in Low Earth Orbit (LEO) and the high latency operations of deep space exploration. The study simulation provided an integrated modeling of a deep space habitat with an environmental control and life support system (ECLSS), an electrical power system (EPS), and a thermal control system (TCS). Simulation interfaces were developed for the NEEMO crew, the mission control support team, and the test conductor. The communications connection between the NEEMO crew interfaces and the mission control support team interfaces had a 15 minute delay to represent the communication delay for a Mars mission.

This study was conducted at NEEMO 21 in year 2016 and was a precursor to more detailed and involved high latency system operation studies in the future. The objective for this year's study was not only to begin to understand the 
interaction between crew and mission control support teams for solving system anomalies across long communication delay, but also to determine the operational environment of NEEMO and optimize hardware for future studies.

A total of 8 subsystem activities were conducted during the NEEMO mission. The result shows that long communication delay significantly increases malfunction reaction time. However, these are preliminary results and subsequent studies will be conducted to better understand and characterize the overall effects of high communications latencies for deep space exploration in general and the interactions between crew and mission control support specifically.

\section{ACKNOWLEDGMENTS}

The authors would like to thank NEEMO $21 \mathrm{crew}$ members, Reid Wiseman, Megan McArthur, Marc O Griofa, Matthias Maurer, Noel Du Toit, and Dawn Kernagis for taking their valuable time to conduct this study, and thank NEEMO mission control team, Moon of Mars team, and NExSyS Team for all their helps and guidance.

\section{REFERENCES}

[1] A. Lin. (2016, Oct) Trick wiki. [Online]. Available: https://github.com/nasa/Trick/wiki

[2] J. Harvey, GUNNS Wiki, L3 Communications, Houston, TX, USA, June 2016.

[3] J. Chambliss, I. Stambaugh, M. Sargusingh, S. Shull, and M. Moore, "Development of a water recovery system resource tracking model," in 45th International Conference on Environmental Systems. Bellevne, Washington: ICES, July 2015.

[4] Space Shuttle Mission STS-128 Press Kit, NASA, August 2009.

[5] S. Loff and B. Dunbar. (2015, July) About neemo nasa extreme environment mission operations. [Online]. Available: http://www.nasa.gov/mission_pages/ NEEMO/about_neemo.html

\section{BIOGRAPHY}

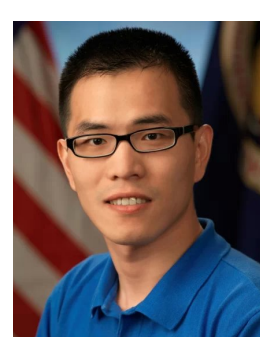

Zu Qun $\mathrm{Li}$ received his B.S degree in aerospace engineering from the Pennsylvania State University in 2012 and a M.S degree in aerospace engineering in 2014. He is currently working in the Simulation and Graphics branch at NASA Johnson Space Center in Houston, Texas. He is also a member of NASA's Exploration Systems Simulation (NExSyS) team working on simulation development and analysis for future human space mission beyond lower Earth orbit.

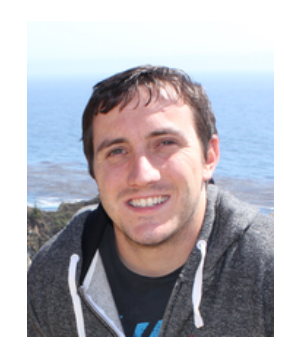

Mike Moore is an aerospace and software engineer for CACI International Inc at Johnson Space Center. He graduated from the University of Texas with a BS in Aerospace Engineering in 2010. Mike has worked for six years at NASA Johnson as a modeling and simulation software engineer. He specializes in spacecraft subsystem simulation. Mike has experience in a wide variety of systems including: guidance, navigation, life support, thermal, and flight control. In his time at JSC, Mike has held several exciting and challenging roles. Most recently, he has served as the lead developer of a guidance, navigation and flight control system simulation for the SpaceX Dragon and Boeing CST-100 vehicles. Outside of NASA, Mike enjoys baseball, soccer, hiking, and hobbyist robotics.

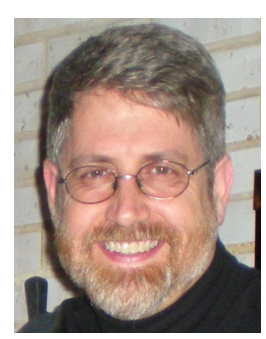

Edwin Z. Crues Edwin Zack Crues has over 25 years of professional experience in developing spacecraft simulation and simulation technologies. Zack is currently a member of the Simulation and Graphics branch at NASAs Johnson Space Center in Houston, Texas (http://er.jsc.nasa.gov/ER7) where he leads the development of simulation technologies and the application of those technologies in the simulation of NASAs current and proposed crewed spacecraft. He has developed hundreds of models and simulations for NASA spacecraft including Shuttle, International Space Station (ISS), Orion, Altair, Morpheus and the Multi-Mission Space Exploration Vehicle. Zacks recent research focus has been developing and applying distributed computation and distributed simulation technologies. This includes a large-scale distributed simulation of NASAs proposed human space exploration missions. Zack also has international experience in developing simulations of European Space Agency launch systems and Japanese Aerospace Exploration Agency spacecraft.

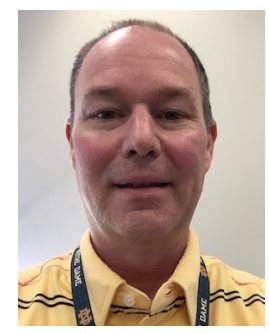

Paul Bielski Paul Bielski is the lead of the NASA Exploration Systems Simulation (NExSyS) team at NASA's Johnson Space Center. After obtaining a B.S. in aerospace engineering from the University of Notre Dame in 1989, he developed spacecraft flight and ground systems for government and commercial entities, worked with international partners to advance on-orbit and groundbased robotic systems, and applied modeling and simulation technology to support analysis, development, test, and operation of existing and future NASA spacecraft. 